\title{
Proteomic analysis of muscle affected by motor neuron degeneration: The wobbler mouse model of amyotrophic lateral sclerosis
}

\author{
Lisa Staunton ${ }^{\mathrm{a}}$, Harald Jockusch ${ }^{\mathrm{b}}$, Kay Ohlendieck ${ }^{\mathrm{a}, *}$ \\ a Department of Biology, National University of Ireland, Maynooth, Co. Kildare, Ireland \\ ${ }^{\mathrm{b}}$ Developmental Biology and Molecular Pathology, University of Bielefeld, Bielefeld, Germany
}

\section{A R T I C L E I N F O}

\section{Article history:}

Received 14 February 2011

Available online 24 February 2011

\section{Keywords:}

Amyotrophic lateral sclerosis

DIGE

Motor neuron disease

Muscular atrophy

Muscle proteomics

Wobbler

\begin{abstract}
A B S T R A C T
Amyotrophic lateral sclerosis is the most common form of motor neuron disease in adult patients and characterized by progressive paralysis. The wobbler mouse (phenotype WR, genotype $w r / w r$ ) is an established animal model of human motor neuron disease and is characterized by a large variety of cellular abnormalities including muscular atrophy. In analogy to recent proteomic studies of cerebrospinal fluid and spinal cord, we have used here fluorescence difference in-gel electrophoresis to analyze global changes in the skeletal muscle proteome from WR versus normal mice. Relative concentrations of 21 proteins were found to be increased and 3 proteins were decreased. Mass spectrometric analysis identified these proteins to be associated with key metabolic pathways, the contractile apparatus, intermediate filaments and the cellular stress response. Drastically increased levels of the glycolytic enzyme glyceraldehyde-3-phosphate dehydrogenase were confirmed by immunoblotting and this finding agrees with the idea of an oxidative-to-glycolytic shift in disease-related muscular atrophy. The establishment of novel disease-specific biomarkers of motor neuron disease might be helpful in the design of improved diagnostic tools and the identification of novel therapeutic targets.
\end{abstract}

(c) 2011 Elsevier Inc. All rights reserved.

\section{Introduction}

Motor neuron diseases are a heterogeneous group of fatal neurological diseases [1]. Disorders with lower motor neuron degeneration are spinal muscular atrophy, an autosomal recessive disorder that is caused by mutations in the survival motor neuron SMN1 gene, spinal bulbar muscular atrophy, a slowly progressive adultonset disorder triggered by the expansion of a polyglutamine tract in the androgen receptor gene and Charcot-Marie-Tooth disease, a diverse group of inherited neuropathies with mutations that cause defects in various neuronal proteins [2]. Amyotrophic lateral sclerosis (ALS), which can be sporadic or be of genetic origin, is the most progressive and most frequent form of adult-onset motor neuron disease [3]. Genetic abnormalities in ALS have been shown to occur in the CuZn-superoxide dismutase encoding SOD1 gene and the TDP-43 encoding TARDBP gene [3]. The degeneration of motor neurons leads to contractile weakness of the limb, bulbar and respiratory muscle systems. Paralysis is highly progressive and is usually fatal due to respiratory failure within 2-3 years for bulbar-onset pathologies and within 3-5 years for limb-onset cases [4]. Currently only one drug, the glutamate antagonist riluzole, has regula-

Abbreviations: 2D-GE, two-dimensional gel electrophoresis; ALS, amyotrophic lateral sclerosis; DIGE, difference in-gel electrophoresis; GAPDH, glyceraldehyde-3phosphate dehydrogenase; MS, mass spectrometry; MyHC, myosin heavy chain.

* Corresponding author. Fax: +353 17083845 .

E-mail address: kay.ohlendieck@nuim.ie (K. Ohlendieck). tory approval for the treatment of ALS, but prolongs the survival time in the treatment of adult-onset motor neuron degeneration by only 3-4 months [5]. Since patients afflicted with ALS have a very poor prognosis, detailed biomedical investigations into the molecular pathogenesis of this fatal neurodegenerative disease and an intensive search for novel therapeutic targets to treat aggressive disorders of the neuromuscular system are warranted.

In this respect, animal models that mimic key symptoms of neurodegeneration and the progressive nature of ALS may be helpful for studying the underlying mechanisms of muscle paralysis [6]. The wobbler mouse (genotype $w r / w r$, phenotype WR) [7] has been used during the last decades as an animal model of motor neuron disease. The recessive mutation $w r$ has been mapped to mouse chromosome 11 [8] and was identified as a missense mutation in the ubiquitously expressed gene Vps54 [9]. The corresponding protein, vesicular protein sorting factor VPS54, is a component of the hetero-trimeric GARP complex involved in retrograde vesicle transport to the Golgi apparatus, and thus plays a crucial part in intracellular transport. The $w r$ allele causes a defect in spermiogenesis [10] in addition to motoneuron degeneration. Knock out of the Vps54 gene leads to embryonic lethality [9]. Recently, it was found that the $w r$ missense mutation destabilizes the VPS54 protein and thereby leads to a reduction of its concentration [11]. Although there is no human neurodegenerative disease known in which the gene for VPS54 (chromosome 2p14-15) is affected [12], the WR mouse with its rapid disease progression nevertheless represents a highly 
valuable model for many aspects of neurodegeneration, including neuroinflammatory processes [13]. The WR mouse is also widely employed for evaluating novel treatment options to counteract symptoms of ALS [14].

Over the last few years, mass spectrometry-based proteomics has been instrumental to perform global surveys of changed protein expression patterns in normal and pathological skeletal muscle tissues [15]. The identification of novel disease-specific biomarker signatures of neuromuscular diseases might be helpful in the design of improved diagnostic tools and the establishment of new therapeutic targets, as well as the superior monitoring of disease progression and determination of adverse effects of experimental drug treatments. The current international effort to discover reliable biomarkers of ALS [16] includes the verification of both suitable metabolites [17] and protein factors [18]. Recent proteomic profiling studies of ALS patient samples and the WR animal model have focused on the analysis of cerebrospinal fluid [19-21] and cervical and lumbar spinal cord [22,23], respectively. The studies on the WR mouse showed that the majority of differentially expressed proteins were related to the glutamate-glutamine cycle, energy transduction, redox functions and astrogliosis [22], thus confirming previous studies [24]. Here, we have applied comparative proteomics to the analysis of skeletal muscle from the WR mouse and established changes in proteins involved in metabolic pathways, the contractile apparatus, intermediate filaments and the cellular stress response.

\section{Materials and methods}

\subsection{Chemicals and materials}

CyDye DIGE Fluor minimal dyes Cy3 and Cy5, Coomassie Blue, ampholytes, cover fluid, acetonitrile and immobilised $\mathrm{pH}$ gradient (IPG) drystrips $\mathrm{pH}$ 3-10 were obtained from Amersham Biosciences/GE Healthcare (Little Chalfont, Buckinghamshire, UK). Sequencing grade-modified trypsin was obtained from Promega (Madison, WI). LC-MS Chromasolv water and formic acid were from Fluka (Milwaukee, WI). Spin filters were obtained from Fisher Scientific (U.K). Protein molecular weight ladders, Laemmli-type buffer and Bradford reagent for protein quantification were purchased from Biorad Laboratories (Hemel-Hempstead, Hertfordshire, UK). Protease inhibitors were from Roche Diagnostics (Mannheim, Germany). Acrylamide stock solutions were obtained as ultrapure Protogel from National Diagnostics (Atlanta, GA). Ultrapure lysine for quenching the DIGE labeling reaction and all general reagents were otained from Sigma Chemical Company (Dorset, UK).

\subsection{Prepartion of muscle extracts from WR and control mice}

The WR mouse represents an established animal model of motor neuron disease [7]. The origin of the WR stock and breeding of mice at the University of Bielefeld has been previously described $[8,9]$. Animals were sacrificed by cervical dislocation and biochemical analyses were performed with post-mortem tissue samples. WR mutants were bred in accordance with German law on the protection of laboratory animals and approved by the local authorities. Tibialis anterior, gastrcocnemius, soleus and vastus lateralis muscles from 9-week old WR mice and their counter-parts from agematched normal C57 mice were freshly dissected and then quick-frozen in liquid nitrogen. WR and control mice had an average body weight of $9.6 \pm 1.6 \mathrm{~g}(n=9)$ and $23.2 \pm 3.0 \mathrm{~g}(n=5)$, respectively. The combined wet weights of the analyzed muscles from both legs of the WR and normal mice were $124 \pm 44 \mathrm{mg}$ $(n=9)$ and $314 \pm 71 \mathrm{mg}(n=5)$, respectively. Samples were transported on dry ice and stored at $-80^{\circ} \mathrm{C}$ prior to usage. For the prep- aration of muscle extracts, tissue specimens were ground, in the presence of liquid nitrogen, into a fine powder using pestle and mortar. For comparative proteomic studies, equal amounts of WR and normal muscle tissue (100 mg wet weight) were used. Muscle powder was suspended in $1 \mathrm{ml}$ of DIGE lysis buffer and urea-soluble proteins extracted as previously described in detail [25].

\subsection{Fluorescence DIGE analysis of muscle extracts}

A $50 \mathrm{mg}$ protein sample was fluorescently labeled for each of the protein samples being studied. Normal muscle extracts and WR muscle extracts were each labeled with Cy3 DIGE Fluor dye and the pooled internal standard with Cy5 DIGE Fluor dye, as previously described in detail [25]. The 2D-GE separation of fluorescently labeled proteins was carried out by using a total of $100 \mathrm{mg}$ protein per DIGE gel. Isoelectric focusing was performed using $24 \mathrm{~cm}$ strips in a IPGphor system from Amersham Biosciences/GE Healthcare (Little Chalfont, Buckinghamshire, UK) using running conditions as previously described [26]. Following firstdimension separation, gel strips were equilibrated for $20 \mathrm{~min}$ in equilibration buffer containing $100 \mathrm{mM}$ dithiothreitol, followed by $10 \mathrm{~min}$ of equilibration in buffer containing $250 \mathrm{mM}$ ideoacetamide. Using the Amersham EttanDalt-twelve system, the gel electrophoretic separation of muscle-associated proteins in the second dimension was performed with standard $12.5 \%(\mathrm{w} / \mathrm{v})$ slab gels [25]. Twelve slab gels were run in parallel at $0.5 \mathrm{~W} /$ gel for 60 minand then $15 \mathrm{~W} /$ gel until the blue dye front had disapeared from the bottom of the gel. Fluorescently labeled proteins were visualized using a Typhoon Trio variable mode imager. Protein expression changes between WR and control samples were analyzed using Progenesis SameSpots analysis software from Non Linear Dynamics (Newcastle upon Tyne, UK). Prior to analysis, individual gels were warped to a single master gel. SameSpots analysis was used to identify proteins of interest using the following parameters: $n=4$; $t$-test $p<0.05$; and a power value of $>0.8$. Proteins with a significantly changed abundance were picked for tryptic digestion from Coomassie Blue-stained preparative gels [25].

\subsection{Mass spectrometric identification of muscle proteins and data verification}

The mass spectrometric identification of proteins of interest was performed with 2-D protein spots from Coomassie-stained pick gels, matched to a DIGE-labeled master gel. Standardized ingel tryptic digestion was used for the generation of peptides and mass spectrometric analysis was carried out on a Model 6430 Ion Trap LC/MS apparatus from Agilent Technologies (Santa Clara, CA). Excision, washing, destaining and treatment with trypsin were performed by previously optimized methods [25]. Peptide samples were dried through vacuum centrifugation and then resuspended in mass spectrometry-grade distilled water and $0.1 \%(\mathrm{v} / \mathrm{v})$ formic acid, spun down through spin filter and added to LC-MS viles for identification by ion trap LC-MS analysis [25]. Separation was performed with a nanoflow Agilent 1200 series system. Samples were loaded into the enrichment at a capillary flow rate set to $2 \mu \mathrm{l} / \mathrm{min}$ with a mix of $0.1 \%(\mathrm{v} / \mathrm{v})$ formic acid and $50 \%(\mathrm{v} / \mathrm{v})$ acetonitrile and formic acid at a ratio of 19:1. The voltage was set to $1700 \mathrm{~V}$. Database searches were carried out with Mascot MS/MS Ion search (Matrix Science, London, UK). For data verification, immunoblotting was carried out as previously described in detail [26].

\section{Results and discussion}

The underlying objective of our proteomic profiling study of the WR mouse was the establishment of global effects of motor neuron 
disease on the protein complement from skeletal muscle tissue. Since conventional protein staining procedures have a limited dynamic range, we employed fluorescence DIGE analysis for the detailed characterization of global changes in the urea-soluble WR muscle proteome. The most prominent changes in gene expression in muscles affected by motor neuron degeneration are expected to reflect physiological denervation. In fact, an increase of the mRNA for the $\alpha$-subunit of the nicotinic acetylcholine receptor, a typical sign of denervation, has been found in WR muscle [27]. In contrast to myotonic mouse muscle, in which there is a shift from the dominant myosin heavy chain (MyHC) isoform IIB (fast-glycolytic) to IIX and IIA (fast-oxidative), in WR muscle there is an opposite shift to an even higher proportion of MyHC IIB and a glycolytic phenotype [28]. In addition, effects related to muscular atrophy are expected. Importantly, since the WR pathology is a long-term and continuous process, as opposed to surgical denervation, intrinsic effects of the partial deficiency in VPS54 may cause proteome-wide alterations in skeletal muscle.

Following differential fluorescent tagging, gel electrophoretic separation, densitometric scanning and image analysis, 24 protein spots were identified in WR muscle tissue that exhibit a significant change in concentration levels. An overview of our DIGE analysis is given in Fig. 1, which illustrates the Cy3 (WR or wild type sample) and Cy5 (pooled standard) fluor labeling approach. A representative master gel is shown for the $\mathrm{pH}$ 3-10 range in Fig. 2. WR-associated changes in the concentration of proteins included twodimensional spots that covered a $\mathrm{p} I$-range from approximately 4.1 (Troponin C) to 8.6 (GAPDH) and ranged in molecular mass from apparent $16 \mathrm{kDa}(\mathrm{Cu} / \mathrm{Zn}$ superoxide dismutase) to $128 \mathrm{kDa}$
(Myosin binding protein C). The findings from the mass spectrometric identification of the 24 protein spots with a changed abundance is presented in Table 1 , listing protein name, accession number, $\mathrm{pI}$-value, molecular mass, number of matched peptide sequences, Mascot score, percentage sequence coverage, Anova score and relative concentrations of individual proteins affected in the WR mouse.

Identified proteins are associated with key metabolic pathways, the contractile apparatus, intermediate filaments and the cellular stress response. Expression levels were reduced in the case of 3 protein species and increased for 21 proteins. The mass spectrometric hit with the highest increase of 6.5 relative concentration is not listed in Table 1 , since this protein was only recognized by 1 peptide (MPSSMGGGGGGSPSPVELR) as histone deacetylase 5 (gi|220941642|, pI 9.07, MW 20,096, Mascot score 28, 10\% coverage, 0.001 Anova). The second highest relative concentration was found for the glycolytic enzyme aldolase (spot 1, Table 1), followed by GAPDH (spot 2). These two enzymes with increased concentrations, in accordance with the previously reported increase in the MyHC isoform IIB [28] reflect a shift towards an extreme glycolytic muscle fiber type. Other increased proteins are represented by actin (spots 4, 9, 16, 19), desmin (spots 5, 7, 11), myoglobin (spots 6 , 12 ), myozenin (spot 8), troponin C (spot 14), actinin (spot 15), $\mathrm{Cu} /$ $\mathrm{Zn}$ superoxide dismutase (spot 20) and fast myosin light chain $1 \mathrm{f}$ (spot 21). Subtypes of creatine kinase M-type, possibly representing differently phosphorylated isoforms, showed differential changes in a variety of protein spots. Five spots exhibited an increased abundance (spots $3,10,13,17,18$ ) and one spot a decreased concentration (spot 22). A decrease was shown for 2
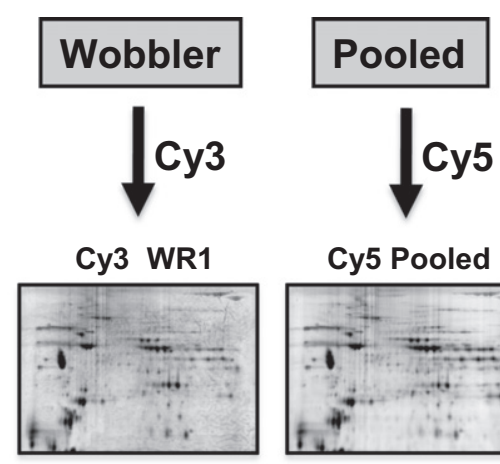

Cy3 WR2

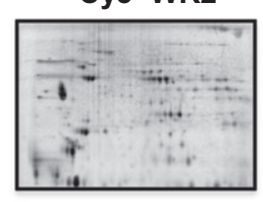

Cy3 WR3

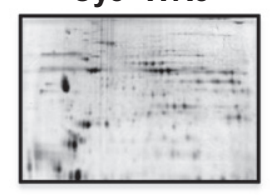

Cy3 WR4

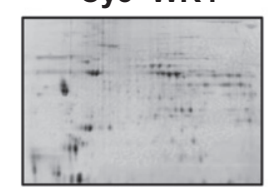

Cy5 Pooled

Cy5 Pooled

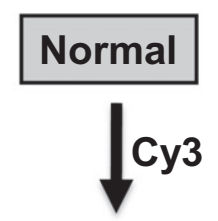

Cy3 WT1

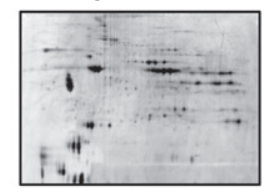

Cy3 WT2
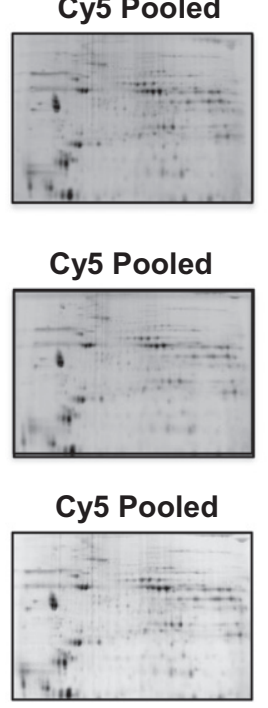

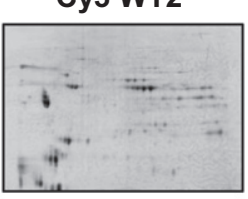

Cy3 WT3

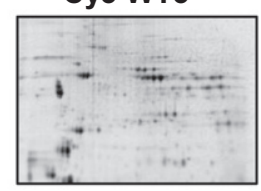

Cy3 WT4

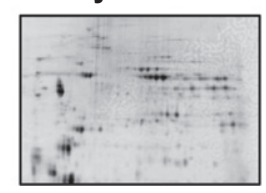

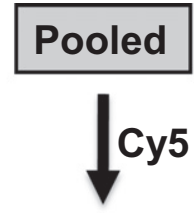

Cy5 Pooled

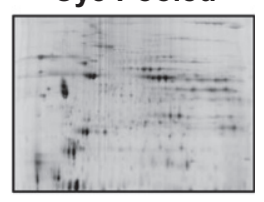

Cy5 Pooled

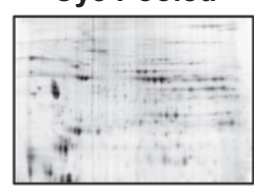

Cy5 Pooled

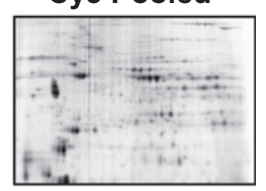

Cy5 Pooled

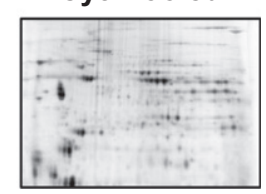

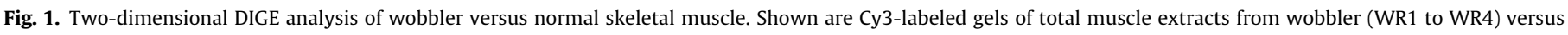
normal wild type (WT1 to WT4) mice, as well as Cy5-labeled gels containing pooled standards. DIGE images are shown for the pH 3-10 range. 


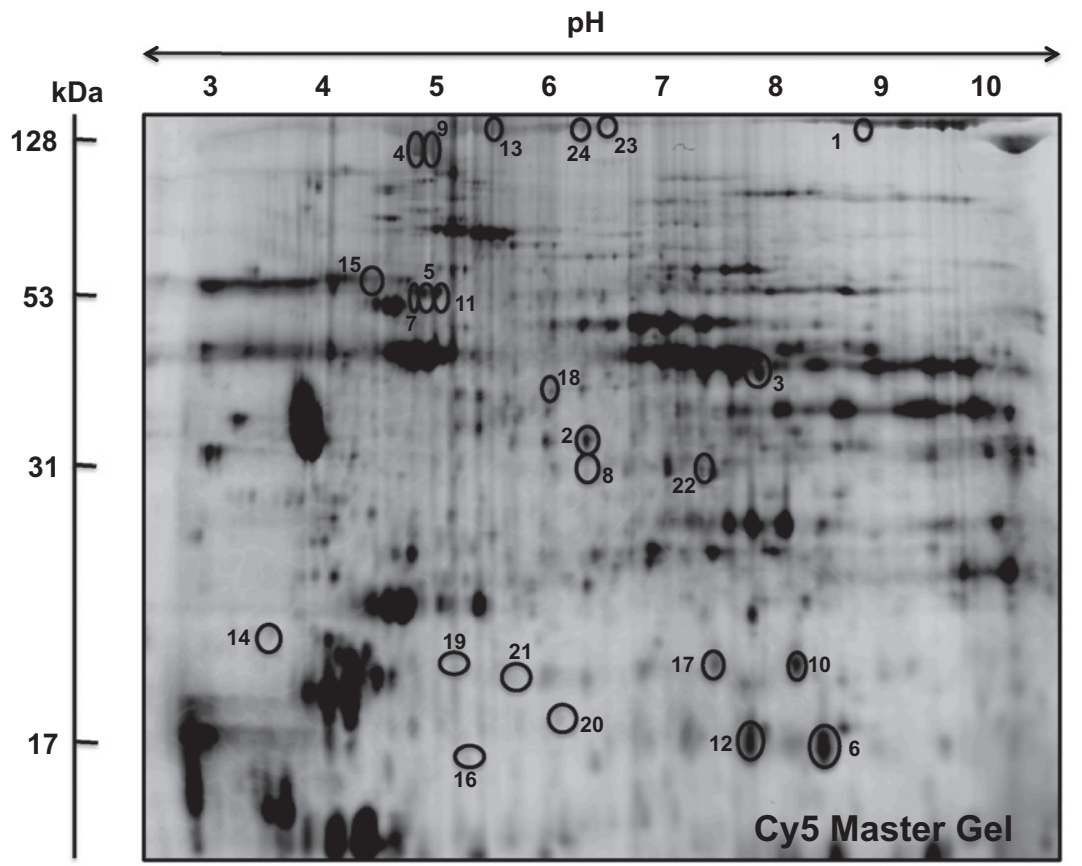

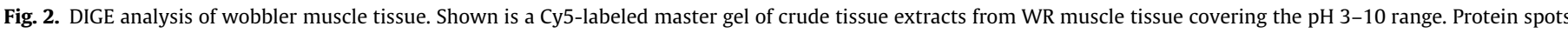

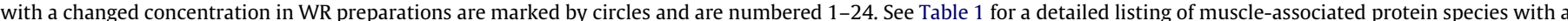

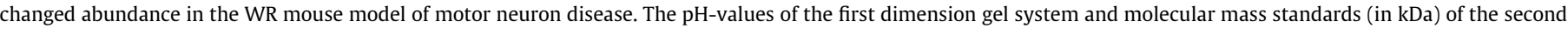
dimension are indicated on the top and on the left of the panels, respectively.

Table 1

DIGE-identified proteins with changed concentration levels in skeletal muscles from the wobbler mouse.

\begin{tabular}{|c|c|c|c|c|c|c|c|c|c|}
\hline $\begin{array}{l}\text { Spot } \\
\text { no. }\end{array}$ & Protein name & Accession No. & $\begin{array}{l}\mathrm{p} I- \\
\text { value }\end{array}$ & $\begin{array}{l}\text { Molecular mass } \\
\text { (Da) }\end{array}$ & $\begin{array}{l}\text { Peptides } \\
\text { matched }\end{array}$ & $\begin{array}{l}\text { Mascot } \\
\text { score }\end{array}$ & $\begin{array}{l}\text { Coverage } \\
(\%)\end{array}$ & Anova & $\begin{array}{l}\text { Fold } \\
\text { change }\end{array}$ \\
\hline 1 & Fructose-bisphosphate aldolase A & gi|6671539| & 8.31 & 39,795 & 2 & 79 & 10 & 0.002 & 4.5 \\
\hline 2 & $\begin{array}{l}\text { Glyceraldehyde-3-phosphate } \\
\text { dehydrogenase }\end{array}$ & gi|149257623| & 8.63 & 36,298 & 6 & 101 & 24 & 0.002 & 3.5 \\
\hline 3 & Creatine kinase M-type & gi|6671762| & 6.58 & 43,250 & 15 & 447 & 41 & 0.006 & 3.4 \\
\hline 4 & Actin, alpha-cardiac & gi|387090| & 5.23 & 42,043 & 12 & 176 & 32 & 0.005 & 3.4 \\
\hline 5 & Desmin & gi|33563250| & 5.21 & 53,523 & 25 & 577 & 57 & 0.003 & 3.3 \\
\hline 6 & Myoglobin & gi|21359820| & 7.07 & 17,117 & 7 & 249 & 59 & 0.007 & 3.2 \\
\hline 7 & Desmin & gi|33563250| & 5.21 & 53,523 & 27 & 475 & 60 & 0.004 & 3 \\
\hline 8 & Myozenin-1 & gi|10946924| & 8.57 & 31,438 & 6 & 159 & 25 & 0.008 & 3 \\
\hline 9 & Actin, alpha-cardiac & gi|387090| & 5.23 & 42,043 & 12 & 185 & 37 & 0.009 & 3 \\
\hline 10 & Creatine kinase M-type & gi|6671762| & 6.58 & 43,250 & 7 & 305 & 20 & 0.011 & 2.8 \\
\hline 11 & Desmin & gi|33563250 & 5.21 & 53,523 & 21 & 369 & 49 & 0.008 & 2.6 \\
\hline 12 & Myoglobin & gi|21359820| & 7.07 & 17,117 & 7 & 274 & 49 & 0.002 & 2.6 \\
\hline 13 & Creatine kinase M-type & gi|6671762| & 6.58 & 43,250 & 5 & 113 & 14 & 0.008 & 2.3 \\
\hline 14 & Troponin C, skeletal muscle & gi|6678371| & 4.07 & 18,156 & 6 & 308 & 49 & 0.005 & 2.2 \\
\hline 15 & Actinin-3, alpha & gi|7304855| & 5.31 & 103,616 & 14 & 330 & 21 & 0.006 & 2.1 \\
\hline 16 & Actin, alpha-cardiac & gi|387090| & 5.32 & 42,048 & 11 & 89 & 28 & 0.013 & 1.9 \\
\hline 17 & Creatine kinase M-type & gi|6671762| & 6.58 & 43,250 & 6 & 102 & 18 & 0.007 & 1.8 \\
\hline 18 & Creatine kinase M-type & gi|6671762| & 6.58 & 43,246 & 7 & 295 & 23 & 0.011 & 1.7 \\
\hline 19 & Actin, alpha & gi|49864| & 5.45 & 38,020 & 8 & 121 & 30 & 0.01 & 1.7 \\
\hline 20 & $\mathrm{Cu} / \mathrm{Zn}$ superoxide dismutase & gi|226471| & 6.03 & 15,926 & 7 & 156 & 47 & 0.005 & 1.6 \\
\hline 21 & Myosin light chain $1 f$ & gi|29789016| & 4.98 & 20,697 & 13 & 187 & 61 & 0.013 & 1.4 \\
\hline 22 & Creatine kinase M-type & gi|6671762| & 6.58 & 43,250 & 10 & 152 & 25 & 0.004 & 0.5 \\
\hline 23 & Myosin binding protein $\mathrm{C}$, fast & gi|20988232| & 6.02 & 128,115 & 14 & 170 & 15 & 0.003 & 0.4 \\
\hline 24 & Myosin binding protein $\mathrm{C}$, fast & gi|20988232| & 6.02 & 128,100 & 22 & 344 & 26 & 0.013 & 0.4 \\
\hline
\end{tabular}

proteins spots which both represent fast myosin binding protein $\mathrm{C}$ (spots 23, 24).

In order to verify key proteomic findings and to put this study into perspective with previous analyses of WR muscle tissues, representative immunoblots are shown in Fig. 3. The previously reported decreased mRNA levels of parvalbumin [27] were shown to correlate to a decreased protein concentration of this cytosolic $\mathrm{Ca}^{2+}$-binding protein. The decrease in parvalbumin and increase in myoglobin on the one hand, and elevated levels of key glycolytic enzymes on the other hand, indicate that the metabolic transformation in WR muscle is more complex than a unidirectional shift towards an extremely glycolytic phenotype. Immunoblotting clearly confirmed our proteomic data and showed a drastically increased abundance of GAPDH and desmin in WR muscle. However, increased levels of $\mathrm{Cu} / \mathrm{Zn}$ superoxide dismutase were not significant. Changes in the concentration of actin, troponin, actinin, 
A Parvalbumin

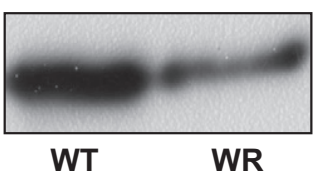

C GAPDH

E Desmin

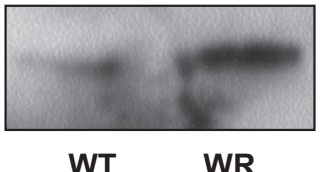

G Cu/Zn SOD

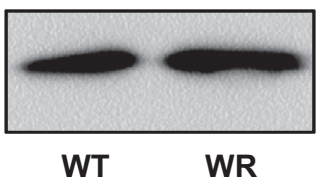

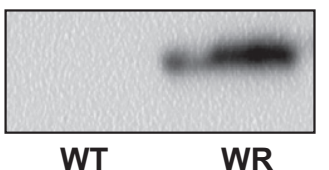
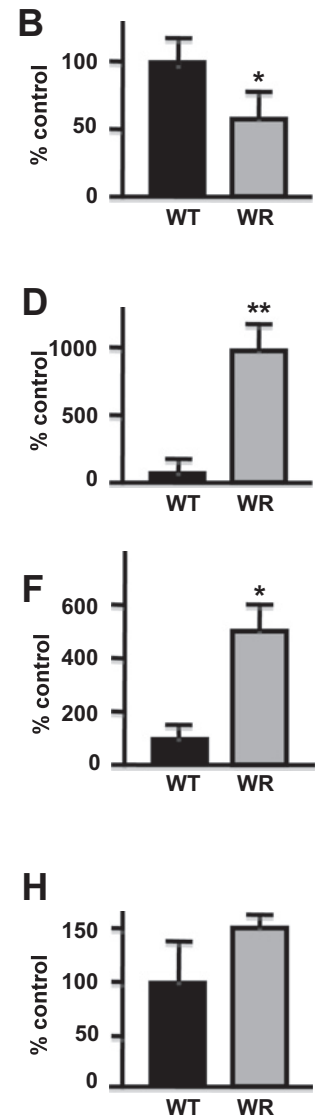

Fig. 3. Immunoblot analysis of wobbler mouse muscle. Shown are representative immunoblots with expanded views of immuno-decorated bands labeled with antibodies to parvalbumin ( $\mathrm{A}$ and $\mathrm{B}$ ), GAPDH ( $\mathrm{C}$ and $\mathrm{D}$ ), desmin ( $\mathrm{E}$ and $\mathrm{F}$ ) and $\mathrm{Cu} / \mathrm{Zn}$ superoxide dismutase (SOD) ( $G$ and $H)$. Lanes 1 and 2 represent normal wild type (WT) versus WR preparations, respectively. The comparative immunoblot analysis was statistically evaluated using an unpaired Student's $t$-test $\left(n=5 ;{ }^{*} p<0.05\right.$; ${ }^{* *} p<0.01$ )

myozenin, myosin light chain and myosin binding protein suggest atrophy-related modulations of the contractile apparatus and the increase in desmin possibly a compensatory stabilization of the cytoskeletal network. The drastic increase in almost all subtypes of creatine kinase imply crucial alterations in creatine phosphate metabolism that might be related to metabolic shifts in WR muscle tissue. Disease-induced atrophy appears to trigger similar slow-tofast transition processes as previously described for disuse atrophy [15]. Diminished neuromuscular activity or the lack of proper loading has distinct effects on the integrity of the skeletal musculature. Immobilization, disuse or denervation triggers muscular atrophy, which clearly shows that neuromuscular innervation patterns are directly linked to gene expression, protein abundance and isoform patterns in skeletal muscles [29].

In analogy to the recent proteomic profiling of cerebrospinal fluids from patients suffering from ALS and the spinal cord from the wobbler mouse [19-23], this study describes the findings of the MS-based proteomic survey of skeletal muscle from the WR mouse. From the detailed analysis of the isoform patterns of the sarcomeric MyHCs it became clear that the response of gene expression in skeletal muscle to denervation is characteristically different from that to hyper-excitability and muscular dystrophy [28]. Thus, studying changes in the WR muscle proteome has the potential to contribute crucial information on disease process during denervation-induced muscular atrophy. Muscular atrophy is associated with a decreased fiber size, a drastic loss in muscle tis- sue mass and a decrease in isometric contractile force. In general, muscular atrophy is observed in skeletal muscles under conditions of microgravity and following extended periods of bed rest, traumatic injury to motor units or pathological damage to motor neurons [29]. Insufficient loading, denervation or disuse is usually associated with slow-to-fast muscle transitions, which has been confirmed by recent MS-based proteomic studies [30-35]. The proteomic profiling of muscular atrophy has revealed an increase in key enzymes of the glycolytic pathway and a concomitant decrease in slow contractile proteins, such as the cardiac isoform of myosin heavy chain, slow troponin T and slow myosin light chain [30-35]. In analogy, the proteomic analysis of the WR mouse, which represents an animal model of disease-induced muscular atrophy, showed an increase in the abundance of key glycolytic enzymes. Thus, the proteomic analysis presented here supports the general idea of disuse- and atrophy-associated oxidative-to-glycolytic transformation processes. In the long-term, the newly identified global changes in the soluble skeletal muscle proteome may be useful candidates for the establishment of a biomarker signature of motor neuron disease [18].

\section{Acknowledgments}

Research was funded by the Irish Health Research Board (HRB$\mathrm{RP} / 2008 / 1)$ ) and Fonds der Chemischen Industrie (FCI). The authors thank Dr. Peter Heimann (Department of Cell Biology, University of Bielefeld, Germany) for his generous help in preparing mutant mouse muscle. The Irish Higher Education Authority and the Deutscher Akademischer Austauschdienst supported several laboratory visits of the Maynooth team in Germany.

\section{References}

[1] C. Wood-Allum, P.J. Shaw, Motor neurone disease: a practical update on diagnosis and management, Clin. Med. 10 (2010) 252-258.

[2] P. Van Damme, W. Robberecht, Recent advances in motor neuron disease, Curr. Opin. Neurol. 22 (2009) 486-492.

[3] L.C. Wijesekera, P.N. Leigh, Amyotrophic lateral sclerosis, Orphanet J. Rare Dis. 4 (2009) 4:3.

[4] R.W. Orrell, Motor neuron disease: systematic reviews of treatment for ALS and SMA, Br. Med. Bull. 93 (2010) 145-159.

[5] R.G. Miller, J.D. Mitchell, M. Lyon, D.H. Moore, Riluzole for amyotrophic lateral sclerosis (ALS)/motor neuron disease (MND), Cochrane Database Syst. Rev. 1 (2007) CD001447.

[6] L.B. Tovar-Y-Romo, L.D. Santa-Cruz, R. Tapia, Experimental models for the study of neurodegeneration in amyotrophic lateral sclerosis, Mol. Neurodegener. 4 (2009) 4:31.

[7] L.W. Duchen, S.J. Strich, An hereditary motor neurone disease with progressive denervation of muscle in the mouse: the mutant 'wobbler', J. Neurol. Neurosurg. Psychiatry 31 (1968) 535-542.

[8] K. Kaupmann, D. Simon-Chazottes, J.L. Guenet, H. Jockusch, Wobbler, a mutation affecting motoneuron survival and gonadal functions in the mouse, maps to proximal chromosome 11, Genomics 13 (1992) 39-43.

[9] T. Schmitt-John, C. Drepper, A. Mussmann, P. Hahn, M. Kuhlmann, C. Thiel, M. Hafner, A. Lengeling, P. Heimann, J.M. Jones, M.H. Meisler, H. Jockusch, Mutation of Vps54 causes motor neuron disease and defective spermiogenesis in the wobbler mouse, Nat. Genet. 37 (2005) 1213-1215.

[10] P. Heimann, S. Laage, H. Jockusch, Defect of sperm assembly in a neurological mutant of the mouse, wobbler (WR), Differentiation 47 (1991) 77-83.

[11] F.J. Perez-Victoria, G. Abascal-Palacios, I. Tascon, A. Kajava, J.G. Magadan, E.P. Pioro, J.S. Bonifacino, A. Hierro, Structural basis for the wobbler mouse neurodegenerative disorder caused by mutation in the Vps54 subunit of the GARP complex, Proc. Natl. Acad. Sci. USA 107 (2010) 12860-12865.

[12] M.H. Meisler, C. Russ, K.T. Montgomery, M. Greenway, S. Ennis, O. Hardiman, D.A. Figlewicz, N.R. Quenneville, E. Conibear, R.H. Brown, Evaluation of the Golgi trafficking protein VPS54 (wobbler) as a candidate for ALS, Amyotroph. Lateral Scler. 9 (2008) 141-148.

[13] J.W. Bartsch, D. Wildeboer, G. Koller, S. Naus, A. Rittger, M.L. Moss, Y. Minai, H. Jockusch, Tumor necrosis factor-alpha (TNF-alpha) regulates shedding of TNFalpha receptor 1 by the metalloprotease-disintegrin ADAM8: evidence for a protease-regulated feedback loop in neuroprotection, J. Neurosci. 30 (2010) $12210-12218$.

[14] K. Iwamoto, Y. Yoshii, K. Ikeda, Atorvastatin treatment attenuates motor neuron degeneration in wobbler mice, Amyotroph. Lateral Scler. 10 (2009) 405-409. 
[15] K. Ohlendieck, Proteomics of skeletal muscle differentiation, neuromuscular disorders and fiber aging, Expert Rev. Proteomics 7 (2010) 283-296.

[16] R. Bowser, M. Cudkowicz, R. Kaddurah-Daouk, Biomarkers for amyotrophic lateral sclerosis, Expert Rev. Mol. Diagn. (2006) 387-398.

[17] S. Rozen, M.E. Cudkowicz, M. Bogdanov, W.R. Matson, B.S. Kristal, C. Beecher, S. Harrison, P. Vouros, J. Flarakos, K. Vigneau-Callahan, T.D. Matson, K.M Newhall, M.F. Beal, R.H. Brown, R. Kaddurah-Daouk, Metabolomic analysis and signatures in motor neuron disease, Metabolomics 1 (2005) 101-108.

[18] H. Ryberg, R. Bowser, Protein biomarkers for amyotrophic lateral sclerosis, Expert Rev. Proteomics 5 (2008) 249-262.

[19] S. Ranganathan, E. Williams, P. Ganchev, V. Gopalakrishnan, D. Lacomis, L Urbinelli, K. Newhall, M.E. Cudkowicz, R.H. Brown, R. Bowser, Proteomic profiling of cerebrospinal fluid identifies biomarkers for amyotrophic lateral sclerosis, J. Neurochem. 95 (2005) 1461-1471.

[20] G.M. Pasinetti, L.H. Ungar, D.J. Lange, S. Yemul, H. Deng, X. Yuan, R.H. Brown, M.E. Cudkowicz, K. Newhall, E. Peskind, S. Marcus, L. Ho, Identification of potential CSF biomarkers in ALS, Neurology 66 (2006) 1218-1222.

[21] H. Ryberg, J. An, S. Darko, J.L. Lustgarten, M. Jaffa, V. Gopalakrishnan, D. Lacomis, M. Cudkowicz, R. Bowser, Discovery and verification of amyotrophic lateral sclerosis biomarkers by proteomics, Muscle Nerve 42 (2010) 104-111.

[22] A. Bastone, E. Fumagalli, P. Bigini, P. Perini, D. Bernardinello, A. Cagnotto, I. Mereghetti, D. Curti, M. Salmona, T. Mennini, Proteomic profiling of cervical and lumbar spinal cord reveals potential protective mechanisms in the wobbler mouse, a model of motor neuron degeneration, J. Proteome Res. 8 (2009) 5229-5240.

[23] J. Zhai, A.L. Strom, R. Kilty, P. Venkatakrishnan, J. White, W.V. Everson, E.J. Smart, H. Zhu, Proteomic characterization of lipid raft proteins in amyotrophic lateral sclerosis mouse spinal cord, FEBS J. 276 (2009) 3308-3323.

[24] S. Laage, G. Zobel, H. Jockusch, Astrocyte overgrowth in the brain stem and spinal cord of mice affected by spinal atrophy, Wobbler. Dev. Neurosci. 10 (1988) 190-198.
[25] K. O'Connell, K. Ohlendieck, Proteomic DIGE analysis of the mitochondriaenriched fraction from aged rat skeletal muscle, Proteomics 9 (2009) 55095524.

[26] C. Lewis, K. Ohlendieck, Proteomic profiling of naturally protected extraocular muscles from the dystrophin-deficient mdx mouse, Biochem. Biophys. Res. Commun. 396 (2010) 1024-1029.

[27] F. Sedehizade, R. Klocke, H. Jockusch, Expression of nerve-regulated genes in muscles of mouse mutants affected by spinal muscular atrophies and muscular dystrophies, Muscle Nerve 20 (1997) 186-194.

[28] O. Agbulut, P. Noirez, G. Butler-Browne, H. Jockusch, Specific isomyosin proportions in hyperexcitable and physiologically denervated mouse muscle FEBS Lett. 561 (2004) 191-194.

[29] S.C. Kandarian, R.W. Jackman, Intracellular signaling during skeletal muscle atrophy, Muscle Nerve 33 (2006) 155-165.

[30] M. Toigo, S. Donohoe, G. Sperrazzo, B. Jarrold, F. Wang, R. Hinkle, E. Dolan, R.] Isfort, R. Aebersold, ICAT-MS-MS time course analysis of atrophying mouse skeletal muscle cytosolic subproteome, Mol. Biosyst. 1 (2005) 229-241.

[31] Z.B. Li, M. Lehar, R. Samlan, P.W. Flint, Proteomic analysis of rat laryngeal muscle following denervation, Proteomics 5 (2005) 4764-4776.

[32] H. Sun, J. Liu, F. Ding, X. Wang, M. Liu, X. Gu, Investigation of differentially expressed proteins in rat gastrocnemius muscle during denervationreinnervation, J. Muscle Res. Cell. Motil. 27 (2006) 241-250.

[33] Y. Seo, R. Lee, K. Park, K. Bae, I. Choi, A proteomic assessment of muscle contractile alterations during unloading and reloading, J. Biochem. 139 (2006) 71-80.

[34] M. Moriggi, P. Cassano, M. Vasso, D. Capitanio, C. Fania, C. Musicco, V. Pesce M.N. Gadaleta, C.A. Gelfi, DIGE approach for the assessment of rat soleus muscle changes during unloading: effect of acetyl-carnitine supplementation, Proteomics 8 (2008) 3588-3604.

[35] R. Ferreira, R. Vitorino, M.J. Neuparth, H.J. Appell, J.A. Duarte, F. Amado, Proteolysis activation and proteome alterations in murine skeletal muscle submitted to 1 week of hindlimb suspension, Eur. J. Appl. Physiol. 107 (2009) 553-563. 